\title{
Clinical Study \\ Sleep Duration and "on" Time during Different Periods of the Day and Night in Patients with Advanced Parkinson's Disease Receiving Adjunctive Ropinirole Prolonged Release
}

\author{
Heinz Reichmann,, ${ }^{1}$ James Cooper, ${ }^{2}$ Katie Rolfe, ${ }^{3}$ and Pablo Martinez-Martinn \\ ${ }^{1}$ Department of Neurology, University of Technology Dresden, Fetscherstraße 74, 01307 Dresden, Germany \\ ${ }^{2}$ Neurosciences Center of Excellence, GlaxoSmithKline, Brentford, London TW8 9GS, UK \\ ${ }^{3}$ Neurosciences Medicines Development Center, GlaxoSmithKline, Uxbridge, London UB11 1BT, UK \\ ${ }^{4}$ Area of Applied Epidemiology, National Centre for Epidemiology and CIBERNED, Carlos III Institute of Health, \\ Madrid 28029, Spain \\ Correspondence should be addressed to Heinz Reichmann, heinz.reichmann@uniklinikum-dresden.de
}

Received 6 December 2010; Revised 28 February 2011; Accepted 6 March 2011

Academic Editor: Peter Hagell

Copyright ( $\odot 2011$ Heinz Reichmann et al. This is an open access article distributed under the Creative Commons Attribution License, which permits unrestricted use, distribution, and reproduction in any medium, provided the original work is properly cited.

\begin{abstract}
Patients undergoing long-term therapy for PD often experience motor fluctuations and nocturnal disturbances. In a post-hoc analysis, we explored effects of ropinirole prolonged release on sleep, night-time awakenings, and "on" time over 24 hours. Patients with advanced PD suboptimally controlled with L-dopa were randomized to adjunctive ropinirole prolonged release (2-24 mg/day) or placebo for 24 weeks. Awake/asleep and, if awake, "on"/"off" status was recorded via diary cards. At week 24 last observation carried forward, changes in nighttime or daytime sleep duration were not significantly different between treatments. Of patients with baseline awakenings, a significantly higher proportion in the ropinirole prolonged release group had a reduction in awakenings versus placebo. Patients receiving ropinirole prolonged release had a significantly greater increase in amount/percentage of awake time "on"/ "on" without troublesome dyskinesia during all periods assessed (including night-time and early morning), versus placebo, and higher odds for being "on" on waking. Adjunctive once-daily ropinirole prolonged release may help provide 24-hour symptom control in patients with advanced PD not optimally controlled with L-dopa.
\end{abstract}

\section{Introduction}

Patients undergoing long-term therapy for Parkinson's disease (PD) often experience motor fluctuations, of which "wearing off" is the first and most frequent complication. This is characterized by impairment of motor control between doses, resulting in periods when symptoms including bradykinesia, rigidity, and tremor are not optimally controlled. A major aim in the management of advanced PD is to decrease motor fluctuations, time spent "off" or "on" with troublesome dyskinesia.

Patients with PD also often suffer from nocturnal disturbances reflected by motor and nonmotor symptoms. Community-based studies report a $60 \%$ prevalence of sleep disorders in patients with PD, compared with 33\% in healthy matched controls $[1,2]$. Insomnia, restless legs syndrome, motor symptoms, pain, nocturia, urinary incontinence, and neuropsychiatric problems are causes of nocturnal sleep disturbances [3]. In a study using a self-completed questionnaire, insomnia was reported by $46 \%$ of patients, restless legs by $42 \%$, acting out during dreams by $36 \%$, intense/vivid dreams by $34 \%$, and daytime sleepiness by $31 \%$ of patients [4]. As a consequence of sleep disorders, patients' quality of life may be seriously impaired $[5,6]$, but this is frequently overlooked in clinical settings [7, 8].

Although treatment with dopamine agonists can alleviate some sleep problems in PD [9], it is thought to be one of the factors contributing to increased daytime sleepiness and sudden onset of sleep [10-12]; preliminary evidence suggests that it may also contribute to sleep fragmentation or night awakenings $[13,14]$. Early morning symptoms of PD may 
be more likely to occur if the effects of the previous dose of dopaminergic medication wear off.

The prolonged-release formulation of the dopamine agonist ropinirole provides patients with continuous ropinirole delivery and fewer fluctuations in plasma levels over 24 hours compared with the immediate-release formulation [15]. Once-daily ropinirole prolonged release is an effective adjunctive therapy in the treatment of PD and has been shown to have beneficial effects on the amount (and percentage) of awake time spent "on" and awake time spent "on" without troublesome dyskinesia [16], as well as on nocturnal symptoms (measured by the Parkinson's Disease Sleep Scale [PDSS]) [16].

Previous analyses of the diary card data collected during EASE-PD Adjunct explored the 24-hour period as a whole to provide total "on"/"off" times [16]. In the current posthoc diary card analyses, the 24-hour period was subdivided in order to assess patients' status during specific periods of the day and night. These analyses were undertaken to explore in more detail the effects of adjunctive ropinirole prolonged release on sleep duration and nighttime awakenings, and to gain better understanding of continued effects over 24 hours by examining "on" time, and "on" time without troublesome dyskinesia, during different periods of the day/night, and the probability of being "on" on waking in the morning.

\section{Methods}

2.1. Study Design and Patients. EASE-PD Adjunct (101468/169; NCT00381472) was a Phase III, randomized, double-blind, parallel-group, placebo-controlled, 24-week, multicentre study that has been described elsewhere [16].

Patients were included if they were aged 30 years or older, had idiopathic PD (Hoehn \& Yahr stage II-IV) not optimally controlled with L-dopa, had been maintained on a stable dose of L-dopa for at least 4 weeks with suboptimal control of symptoms, and had spent at least 3 hours/day awake time "off" during the baseline period. Exclusion criteria included: incapacitating dyskinesia, any dopamine agonist use within 4 weeks of screening, significant or uncontrolled psychiatric, neurological or other medical disorders, dementia, drug abuse or alcoholism. All patients provided written informed consent approved by their respective ethics committee or institutional review board. The study was conducted in accordance with good clinical practice, patient confidentiality requirements, and the guiding principles of the Declaration of Helsinki.

2.2. Treatment. Patients were randomized $(1: 1)$, using a computerized schedule, to 24 weeks of treatment with adjunctive ropinirole prolonged release or placebo, concurrent with their pre-existing L-dopa treatment. To ensure blinding, the titration schedule referred to numbered dosage levels of study medication, both groups were dosed similarly, and ropinirole prolonged release and placebo tablets were identical in appearance and packaging. Titration and adjustment of both study medication and L-dopa were based on the individual clinician's judgment.
The starting dose of study medication was $2 \mathrm{mg} /$ day, and doses were to be titrated to the optimal therapeutic dose, up to a maximum of $24 \mathrm{mg} /$ day. When a dose of $8 \mathrm{mg} /$ day of study medication was reached, the dose of L-dopa was to be reduced and reduced again with each subsequent increase in study medication. If symptom control was lost, the dose of study medication was to be increased to the next dose level. If symptoms did not improve after two up-titrations of study medication, the L-dopa dose could be increased up to (but not above) the baseline dose.

Selegiline, amantadine, anticholinergics, and catechol-Omethyl-transferase inhibitors were permitted, provided the dose was stable for at least 4 weeks prior to screening and remained stable during the study.

2.3. Assessments. Patients were evaluated at baseline and weeks $1,2,3,4,6,8,10,12,16,20$, and 24. Diary cards (Figure 1) were used to record whether patients were awake or asleep, and whether they were "on" or "off" (if awake), and, if "on", whether they experienced troublesome dyskinesia for each 0.5 -hour period during two 24-hour periods. Patients were asked to complete two 24-hour diary cards prior to each postbaseline study visit. During the 2 weeks of the placebo run-in period, patients were asked to complete two 24-hour diary cards during each week on the same 2 days of each relevant week.

Previous analyses of diary cards explored the 24-hour period as a whole (using the mean of two 24-hour diaries) to provide total "on"/"off" times [16]. In the current post-hoc analyses, the 24-hour period was subdivided, with the 0.5hour periods grouped into different time intervals, defined retrospectively as: nighttime, $10 \mathrm{pm}-8 \mathrm{am}$; daytime, $8 \mathrm{am}-$ $10 \mathrm{pm}$; early morning, $5 \mathrm{am}-10 \mathrm{am}$; morning, $8 \mathrm{am}-1 \mathrm{pm}$; afternoon, $1 \mathrm{pm}-6 \mathrm{pm}$; and evening, $6 \mathrm{pm}-10 \mathrm{pm}$.

Diary card entries were used to assess the duration/proportion of sleep time, number of nighttime awakenings, "off" and "on" periods, proportion of patients "on" on waking, and time spent "on" without troublesome dyskinesia in both groups.

\subsection{Statistical Analyses}

2.4.1. Endpoints. Changes from baseline analyzed post-hoc at week 24 last observation carried forward (LOCF) were: the duration of sleep during the day, morning, afternoon, evening, and nighttime; the amount of awake time spent "on", and "on" without troublesome dyskinesia, during the day, morning, afternoon and evening; and the percentage of awake time spent "on", and "on" without troublesome dyskinesia, during the night and early morning.

The proportion of patients with a decrease from baseline in the number of awakenings per night and the proportion of patients "on" on waking in the morning (according to both diary days) at week 24 LOCF were also analyzed post-hoc.

2.4.2. Analyses. Patients were considered "on" on waking at a visit if they were "on" on waking for both diary days otherwise; they were considered "off". Due to the low 


\begin{tabular}{|c|c|c|c|c|c|}
\hline Protocol number & Subject number & Day & $\begin{array}{l}\text { Date } \\
\text { Month }\end{array}$ & Year & Visit description \\
\hline $101468 / 169$ & & & & & Week-2 \\
\hline
\end{tabular}

Complete this page on the first day

\begin{tabular}{|c|c|c|c|c|c|c|c|c|c|}
\hline $\begin{array}{c}\text { Time } \\
\text { AM }\end{array}$ & Sleep & OFF & ON & $\begin{array}{c}\text { Troublesome } \\
\text { dyskinesias during this } \\
\text { ON time }\end{array}$ & $\begin{array}{l}\text { Time } \\
\text { PM }\end{array}$ & Sleep & OFF & $\mathrm{ON}$ & $\begin{array}{c}\text { Troublesome } \\
\text { dyskinesias during this } \\
\text { ON time }\end{array}$ \\
\hline $\begin{array}{c}\text { 12:00 } \\
\text { Midnight }\end{array}$ & & & & & $\begin{array}{l}12: 00 \\
\text { Noon }\end{array}$ & & & & \\
\hline $12: 30$ & & & & & $12: 30$ & & & & \\
\hline 1:00 & & & & & $1: 00$ & & & & \\
\hline $1: 30$ & & & & & $1: 30$ & & & & \\
\hline 2:00 & & & & & 2:00 & & & & \\
\hline $2: 30$ & & & & & $2: 30$ & & & & \\
\hline $3: 00$ & & & & & $3: 00$ & & & & \\
\hline $3: 30$ & & & & & $3: 30$ & & & & \\
\hline $4: 00$ & & & & & $4: 00$ & & & & \\
\hline $4: 30$ & & & & & $4: 30$ & & & & \\
\hline $5: 00$ & & & & & $5: 00$ & & & & \\
\hline $5: 30$ & & & & & $5: 30$ & & & & \\
\hline $6: 00$ & & & & & $6: 00$ & & & & \\
\hline $6: 30$ & & & & & $6: 30$ & & & & \\
\hline $7: 00$ & & & & & $7: 00$ & & & & \\
\hline $7: 30$ & & & & & $7: 30$ & & & & \\
\hline $8: 00$ & & & & & $8: 00$ & & & & \\
\hline $8: 30$ & & & & & $8: 30$ & & & & \\
\hline 9:00 & & & & & $9: 00$ & & & & \\
\hline $9: 30$ & & & & & $9: 30$ & & & & \\
\hline $10: 00$ & & & & & $10: 00$ & & & & \\
\hline $10: 30$ & & & & & $10: 30$ & & & & \\
\hline $11: 00$ & & & & & $11: 00$ & & & & \\
\hline $11: 30$ & & & & & $11: 30$ & & & & \\
\hline
\end{tabular}

FIgURe 1: Blank diary card. 
TABLE 1: Demographics and baseline characteristics of patients (intent-to-treat population).

\begin{tabular}{|c|c|c|}
\hline & Ropinirole prolonged release $(n=201)$ & Placebo $(n=190)$ \\
\hline Age, years & $66.3(9.17)$ & $66.0(9.74)$ \\
\hline Women, $n(\%)$ & $84(42)$ & $61(32)$ \\
\hline Age at onset of PD, years & $57.6(10.53)^{\mathrm{a}}$ & $57.3(10.74)^{\mathrm{b}}$ \\
\hline Duration of PD, years & $8.6(4.76)^{\mathrm{a}}$ & $8.6(5.15)^{\mathrm{b}}$ \\
\hline Duration of L-dopa treatment, years & $6.5(4.45)^{\mathrm{c}}$ & $6.6(4.33)^{\mathrm{d}}$ \\
\hline L-dopa dose, mg/day & $824(424.4)^{c}$ & $776(357.3)$ \\
\hline Total daily awake time spent "on", hours & $9.0(2.75)$ & $9.1(2.69)$ \\
\hline \multicolumn{3}{|l|}{ Amount of awake time spent "on", hours } \\
\hline Daytime (8 am-10 pm) & $7.9(2.24)^{\mathrm{e}}$ & $7.8(2.13)^{f}$ \\
\hline Morning ( 8 am-1 pm) & $3.0(1.06)^{\mathrm{e}}$ & $2.9(0.97)^{\mathrm{f}}$ \\
\hline Afternoon (1 pm-6 pm) & $2.7(0.90)^{e}$ & $2.7(0.87)^{\mathrm{f}}$ \\
\hline Evening $(6 \mathrm{pm}-10 \mathrm{pm})$ & $2.2(0.93)^{e}$ & $2.2(0.95)^{\mathrm{f}}$ \\
\hline \multicolumn{3}{|l|}{ Percentage awake time spent "on" } \\
\hline Nighttime (10 pm-8 am) & $40.0(25.79)^{\mathrm{e}}$ & $39.9(28.49)^{\mathrm{f}}$ \\
\hline Early morning ( $5 \mathrm{am}-10 \mathrm{am})$ & $50.2(25.20)^{\mathrm{e}}$ & $47.6(24.99)^{\mathrm{f}}$ \\
\hline Patients "on" on waking in the morning, $n(\%)^{g}$ & $28(15)^{\mathrm{d}}$ & $24(14)^{\mathrm{h}}$ \\
\hline \multicolumn{3}{|l|}{ Duration of sleep time, hours } \\
\hline Daytime (8 am-10 pm) & $0.8(0.99)^{\mathrm{e}}$ & $0.9(1.07)^{\mathrm{f}}$ \\
\hline Nighttime (10 pm-8 am) & $7.1(1.48)^{\mathrm{e}}$ & $7.1(1.31)^{\mathrm{f}}$ \\
\hline Patients with no awakenings/night, $n(\%)^{\mathrm{g}}$ & $110(59)^{\mathrm{d}}$ & $86(50)^{\mathrm{h}}$ \\
\hline
\end{tabular}

${ }^{\mathrm{a}} n=200 ;{ }^{\mathrm{b}} n=188 ;{ }^{\mathrm{c}} n=199 ;{ }^{\mathrm{d}} n=187 ;{ }^{\mathrm{e}} n=190 ;{ }^{\mathrm{f}} n=175 ;{ }^{\mathrm{g}}$ week 0 data (two diary card days from the second week of the baseline period)), ${ }^{\mathrm{h}} n=173$. All values are mean (SD) unless stated otherwise.

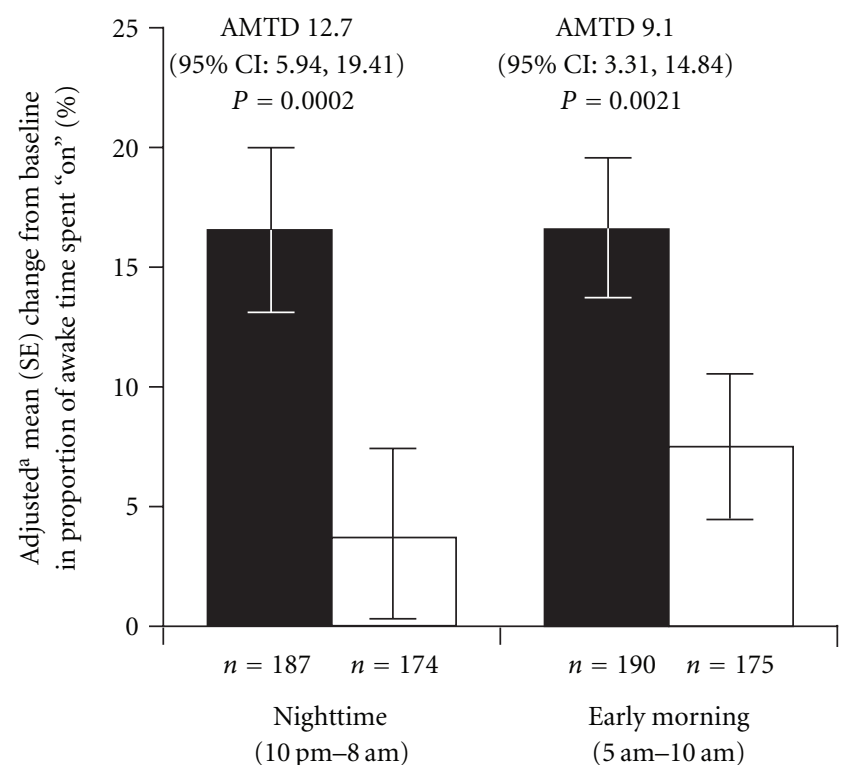

Ropinirole prolonged release

Placebo

AMTD, adjusted mean treatment difference;

$\mathrm{CI}$, confidence interval; SE, standard error

aAdjusted for country and baseline proportion of awake time spent "on" (\%)

Figure 2: adjusted mean change from baseline at week 24 LOCF in the proportion of awake time spent "on" during the night and in the early morning (intent-to-treat population).

proportion of patients waking "on" at baseline according to this definition, a sensitivity analysis was also performed whereby patients were considered "on" if they were "on" on waking on either of the diary days. A nighttime awakening was defined as a period during the night $(10 \mathrm{pm}-8 \mathrm{am})$ in which patients recorded themselves as "on" or "off" (one or more consecutive 0.5 -hour periods awake, regardless of state), preceded and succeeded by a recorded sleep time. For assessments of the number of awakenings per night, the maximum number of awakenings from the two diary days at each study visit was used for analysis.

The intent-to-treat population (all patients who received at least one dose of study medication and who had at least one postbaseline efficacy assessment) was used for all analyses. For continuous endpoints, baseline was taken as the mean of the four 24-hour periods recorded between screening and baseline; however, for analyses of being "on" on waking, and number of nighttime awakenings, baseline measurements utilized only the two diary cards from the second week (i.e., week 0 data) to ensure methodology was consistent with that used for postbaseline data. All data for postbaseline visits were calculated using the mean of the two diary days completed before each visit. Changes from baseline to week 24 LOCF were analyzed for between-group differences using an analysis of covariance (ANCOVA) model, adjusted for country and baseline value; if model assumptions underlying the parametric analysis were not respected, a nonparametric analysis (van Elteren test), adjusted for country, was utilized. A logistic regression model, adjusting for country, was used to analyze the proportion of patients with a decrease from 


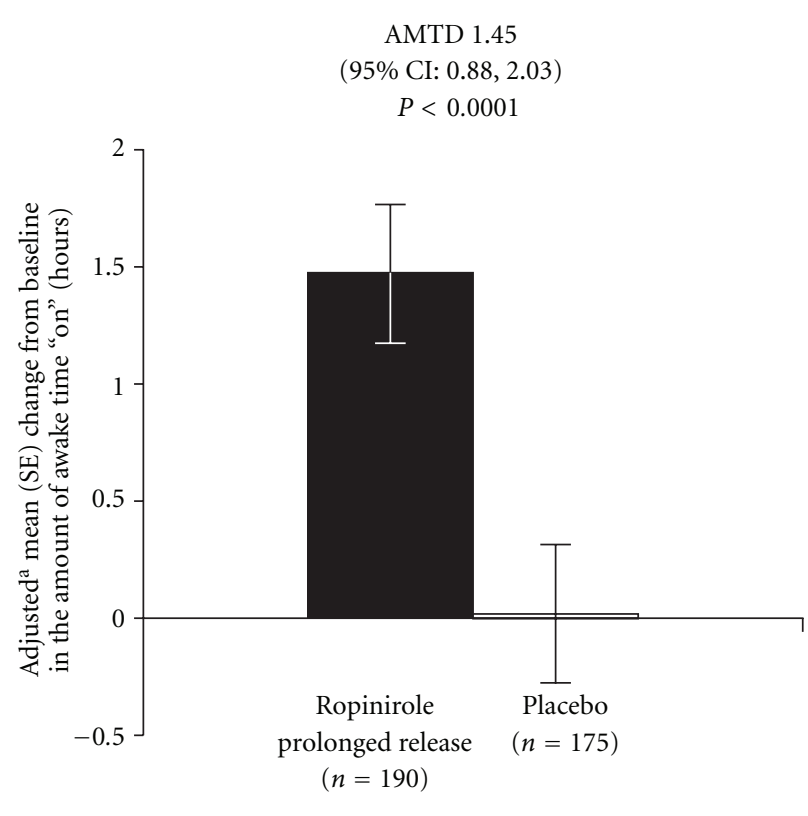

(a)

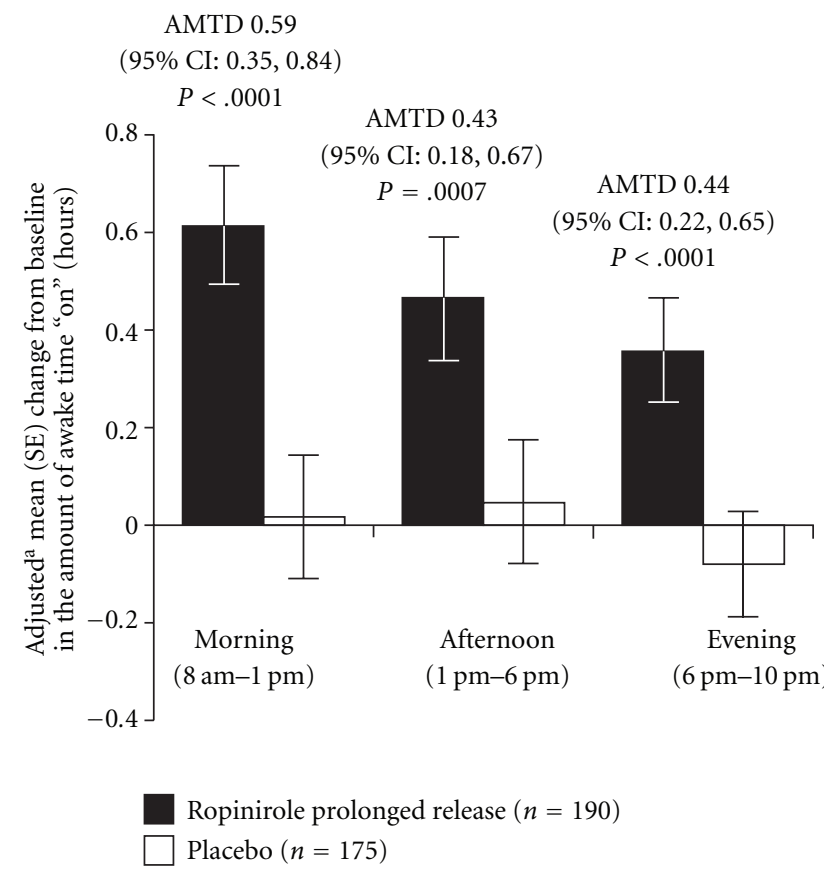

AMTD, adjusted mean treatment difference; $\mathrm{CI}$, confidence interval; LOCF, last observation carried forward; SE, standard error.

aAjusted for country and baseline amount of awake time "on" (hours).

(b)

Figure 3: aAjusted mean change from baseline in the amount of awake time spent "on" (hours) at week 24 LOCF (intent-to-treat population) (a) during the daytime ( $8 \mathrm{am}-10 \mathrm{pm})$; (b) during each period of the daytime.

baseline in the number of awakenings per night, and the proportion of patients "on" on waking in the morning, at week 24 LOCF. Missing data were handled using LOCF imputation.

\section{Results}

3.1. Patients. Baseline characteristics are presented in Table 1. Diary card data were unavailable for 26/391 patients at baseline (11 ropinirole prolonged release, 15 placebo), and 24/391 patients at week 24 LOCF (11 ropinirole prolonged release, 13 placebo). Therefore, analyses included 190 patients in the ropinirole prolonged release group and 175 patients in the placebo group. As reported previously [16], 191/201 (95\%) patients in the ropinirole prolonged release group and 174/190 (92\%) in the placebo group had Ldopa dose reduction; reinstatement of L-dopa following dose reduction was required for $14 / 191$ (7\%) and 49/174 (28\%) patients, respectively. The reduction in L-dopa dose was larger in the ropinirole prolonged release group than in the placebo group (mean standard deviation (SD) change from baseline -278 [193] mg/day versus - 164 [164] mg/day).

3.2. Sleep Duration during the Night and Day. At baseline, the mean (SD) amount of sleep time during the night and day was similar for patients in both groups (Table 1). At week 24 LOCF, the mean (SD) duration of sleep time during the nighttime and daytime was 7.34 (1.47) and 0.9 (1.15) hours for patients in the ropinirole prolonged release group, and 7.22 (1.50) and 1.0 (1.24) hours in the placebo group, respectively.

At night, sleep duration increased in both groups from baseline to week 24 LOCF, and there was a nonsignificant trend for a greater increase in the ropinirole prolonged release group (adjusted mean treatment difference [AMTD], 0.16 ; 95\% confidence interval $[\mathrm{CI}]:-0.09,0.41 ; P=0.2041)$. Both treatment groups had a small increase in sleep duration (approximately 6-7 minutes) during the daytime but there was no significant difference between groups $(P=0.4205$; nonparametric van Elteren test). There were small changes from baseline for individual periods of the day, which were nonsignificant between treatments.

3.3. Nocturnal Awakenings. No significant treatment effect was detected for the proportion of patients with a decrease from baseline in the number of awakenings per night at week 24 LOCF (odds ratio 1.43; 95\% CI: 0.85, 2.39; $P=$ $0.180)$, although a higher proportion of patients receiving ropinirole prolonged release $(25 \% ; 46 / 187)$ had a decrease compared with placebo $(18 \% ; 32 / 173)$. These relatively small proportions may be due to a high proportion of patients in each group (110/187 [59\%] ropinirole prolonged release, 86/173 [50\%] placebo) having no awakenings per night at baseline (Table 1) according to the definition applied. An analysis excluding the patients with no baseline nocturnal awakenings showed a larger treatment effect, with a higher proportion of patients receiving ropinirole prolonged release $(60 \% ; 46 / 77)$ having a decrease from baseline in the number of awakenings per night at week 24 LOCF compared with placebo $(37 \% ; 32 / 87)$. This difference was significant (adjusted [for country] odds ratio 2.46; 95\% CI 1.29, 4.68; $P=0.0060)$. 


\subsection{Motor Symptom Control}

3.4.1. Awake Time Spent "on" during the Nighttime and Early Morning. At week 24 LOCF, mean (SD) percentage of awake time spent "on" was: $57.0 \%$ (32.22) at nighttime and 64.5\% (29.27) in the early morning for the ropinirole prolonged release group, and $43.6 \%$ (37.44) at nighttime and $53.7 \%$ (31.31) in the early morning for the placebo group. Patients receiving ropinirole prolonged release had a significantly greater increase in the percentage of awake time spent "on" both during the nighttime (AMTD 12.7\%; 95\% CI: 5.94, $19.41 ; P=0.0002$ ) and early morning (AMTD 9.1\%; 95\% CI: $3.31,14.84 ; P=0.0021)$, compared with patients receiving placebo (Figure 2 ). There were also statistically significant treatment benefits in favor of ropinirole prolonged release for the percentage of awake time spent "on" without troublesome dyskinesia, at week 24 LOCF for the nighttime and early morning. Compared with placebo, patients who received ropinirole prolonged release had, on average, $11 \%$ more awake time spent "on" without troublesome dyskinesia during the night (AMTD: 10.94; 95\% CI: 4.21, 17.66; $P=$ $0.0015)$ and $8 \%$ more during the early morning (AMTD: 8.10; 95\% CI: 2.28, 13.93; $P=0.0065)$.

3.4.2. Awake Time Spent "on" during the Daytime. At baseline, the mean (SD) amount of awake time spent "on" during the daytime $(8 \mathrm{am}-10 \mathrm{pm})$ was similar (approximately 8 hours) for patients receiving ropinirole prolonged release and placebo (Table 1).

At week 24 LOCF, the mean (SD) amount of awake time spent "on" during the daytime was 9.4 (3.09) hours for patients receiving ropinirole prolonged release and 7.9 (3.16) hours for patients receiving placebo.

There was a significantly greater improvement from baseline to week 24 LOCF in "on" time while awake during the daytime for patients receiving ropinirole prolonged release versus placebo (AMTD: 1.45; 95\% CI: 0.88, 2.03; $P<0.0001$; Figure $3(\mathrm{a}))$. For each of the daily time periods considered, there were statistically significant treatment benefits in favor of ropinirole prolonged release for the change from baseline in the amount of awake time spent "on", at week 24 LOCF (Figure 3(b)).

There were also statistically significant treatment benefits in favor of ropinirole prolonged release for time spent "on" without troublesome dyskinesia, at week 24 LOCF for the daytime (AMTD: 1.29; 95\% CI: $0.71,1.87 ; P<0.0001$ ), and for each of the different daily periods considered: morning (AMTD: 0.54; 95\% CI: 0.29, 0.78; $P<0.0001$ ), afternoon (AMTD: $0.40 ; 95 \% \mathrm{CI}: 0.15,0.64 ; P=0.0016)$, and evening (AMTD: 0.39; 95\% CI: 0.17, $0.60 ; P=0.0004$ ).

3.4.3. Probability of Waking "on". Despite the stringent definition applied for being "on" ("on" on waking for both diary days), over one-third (37\%; 70/190) of patients receiving ropinirole prolonged release awoke "on" at week 24 LOCF, compared with $26 \%(46 / 177)$ in the placebo group. For a patient receiving ropinirole prolonged release, the adjusted (for country) odds of being "on" on waking in the morning were 1.65 (95\% CI: $1.05,2.59)$ times higher than for a patient receiving placebo, and this difference was significant $(P=0.029)$. A sensitivity analysis with a less stringent definition ("on" on waking on either day) showed that a higher proportion of patients in both groups awoke "on" at week 24 LOCF (52\% [98/190] for ropinirole prolonged release and $36 \%$ [63/177] for placebo) and this supported the original analysis (adjusted odds of being "on" on waking in the morning, 1.94 [95\% CI: $1.27,2.96$ ]; $P=0.002$ ).

\section{Discussion}

Ropinirole prolonged release is a non-ergoline D2/D3 dopamine agonist which has been shown to be effective in PD not only when used as monotherapy [17], but also when used as an adjunct to L-dopa in advanced PD [16]. In addition to improvement in motor fluctuations in this patient population, treatment with ropinirole prolonged release has been shown to permit a reduction in L-dopa dose (approximately 30\% [16]) and this may help to ameliorate the dyskinesia that can emerge upon initiation of adjunctive therapy. Initial analysis of the EASE-PD Adjunct study showed that once-daily ropinirole prolonged release as an adjunct to L-dopa increased "on" time over a 24-hour period in patients with advanced $\mathrm{PD}$, and also reduced nocturnal symptoms (as measured by the PDSS) [16]. Data from this study have also shown that benefits are seen as early as 2 weeks after treatment initiation [18]. The post-hoc analyses of the diary card data from EASE-PD reported here show that significant improvements in "on" time were experienced during the nighttime and in all periods of the day by patients receiving ropinirole prolonged release, compared with placebo, despite only once-daily dosing. This was not due to a decrease in overall sleep duration, which remained similar between treatment groups. Patients receiving ropinirole prolonged release also had a significantly higher probability of being "on" on waking relative to those receiving placebo. These data confirm that once-daily treatment with ropinirole prolonged release is capable of providing consistent symptomatic benefits throughout the whole day.

To our knowledge, this is the first analysis in which the 24-hour dose period has been subdivided into specific periods to assess the continued effect of prolonged-release dopamine agonist treatment. This allowed the determination of whether symptomatic benefits were consistently observed across different periods of the day and night and enabled the study of quantitative aspects of sleep not captured by the PDSS.

No significant treatment differences were observed in change in nighttime or daytime sleep duration. Overall, nighttime and daytime sleep increased slightly during the study, but changes were small and similar for both groups. The data from this study suggest that ropinirole prolonged release neither significantly reduces nighttime sleep nor increases sleep time during the day in patients with advanced PD. This latter point is consistent with previously reported 
nonsignificant differences in change in Epworth Sleepiness Scale scores from this study [16].

In the placebo group, $18 \%$ of patients experienced a decrease in the number of awakenings per night from baseline, compared with $25 \%$ in the ropinirole prolonged release group; this was not statistically significant, possibly because a high proportion of patients in both groups (59\% ropinirole prolonged release, $50 \%$ placebo) had no awakenings at night at baseline (and therefore could not experience a reduction). This position is supported by the results of a further analysis which excluded those patients with no awakenings at night at baseline; this showed that a significantly higher proportion of patients in the ropinirole prolonged release group (60\%) versus the placebo group (37\%) experienced a decrease in the number of awakenings from baseline.

Over 24 weeks, patients receiving ropinirole prolonged release gained almost 1.5 hours more awake time "on" during the day $(8 \mathrm{am}-10 \mathrm{pm})$ than patients receiving placebo. A significant benefit for ropinirole prolonged release was seen during the morning, and these effects were maintained throughout the afternoon and evening. Results for awake time spent "on" without troublesome dyskinesia were supportive of benefits across the night and daytime periods, consistent with results reported for the 24 -hour period as a whole [16].

As reported in the main analysis, ropinirole prolonged release was well tolerated in the patient population studied, and adverse events reported were typical for a dopamine agonist [16]. However, when considering treatments with these medications, including ropinirole prolonged release, it is important to be mindful of the precautions associated with this class, including impulse control disorders and episodes of sudden onset of sleep [19], and take these into consideration along with the potential benefits.

Self-reported diary cards are important tools in clinical research [20], as they provide an accurate reflection of clinical status over a period of time and can generate a lot of information in a simple format. However, there may be limitations associated with the data collected. Responses are subjective, and therefore the validity of the data relies on patients accurately completing their diaries, which may, in turn, be influenced by the patient's mental status; for example, decreasing memory over time may affect patients' ability to complete diary cards in a timely manner. With no indication of the timing of diary completion in this study, this parameter could not be assessed. Another potential limitation is that due to the way the diary is structured, data are collected in 30-minute increments. Thus, results may be insensitive to exact timing of events, for example, for falling asleep or waking up: defined time periods may not accurately reflect each individual patient's sleep patterns. Measuring nighttime awakenings in this way is likely to be particularly insensitive; use of polysomnography, actigraphy or even subjective reports would most probably have identified many more awakenings than were captured by the diary card approach. To our knowledge, no such studies have been conducted on a large scale with ropinirole prolonged release, although some findings have been reported in a subset of patients from a small, unblinded trial [21]. In addition, on diary days, the need to complete the diary card in an accurate and timely fashion may cause patients undue worry, which may affect their usual routine. Finally, it must be acknowledged that the analyses presented here were performed post-hoc, and therefore a degree of caution should be exercised when interpreting the findings. Despite methodological differences, the results of these post-hoc analyses are in line with those from the main analysis of the trial [16].

\section{Conclusion}

Although post-hoc, this novel analysis of diary card data provides additional support that once-daily ropinirole prolonged release as an adjunctive therapy may help to provide 24-hour symptom control in patients with advanced PD not optimally controlled by L-dopa. Patient benefits are apparent during the day and night, and immediately upon waking. As the population of patients included in this controlled study appears to be representative of patients with advanced stage PD seen in daily practice, the results can be considered relevant and applicable to a real-life setting.

\section{Acknowledgments and Disclosures}

This study was supported by GlaxoSmithKline (GSK) R\&D and SkyePharma. The authors would like to thank Kirsty Rayner (GSK) for statistical review. H. Reichmann has acted on Advisory Boards and given lectures for, and received research grants from, Bayer Health Care, BoehringerIngelheim, Cephalon, Desitin, GSK, Merck-Serono, Novartis, Orion, Pfizer, Solvay, TEVA/Lundbeck, UCB Schwarz Pharma, and Valeant. P. Martinez-Martin has served as an independent scientific consultant for Novartis Pharmaceutical Spain, UCB, and GSK, and as a speaker in sponsored events by Newron, Boehringer-Ingelheim, Novartis, GSK, UCB, Solvay, and Cephalon. He has received research support from the Michael J. Fox Foundation (USA), Carlos III Institute of Health and CIBERNED (Spain). J. Cooper and K. Rolfe are employees in, and stockholders in, GSK. Editorial support in the form of writing an outline and initial draft of the manuscript, drafting tables and figures, and collating author comments, was provided by Joanna Wright, DPhil (Caudex Medical, Oxford, UK), funded by GSK.

\section{References}

[1] E. Tandberg, J. P. Larsen, and K. Karlsen, "Excessive daytime sleepiness and sleep benefit in Parkinson's disease: a community-based study," Movement Disorders, vol. 14, no. 6, pp. 922-927, 1999.

[2] K. Karlsen, J. P. Larsen, E. Tandberg, and K. Jørgensen, "Fatigue in patients with Parkinson's disease," Movement Disorders, vol. 14, no. 2, pp. 237-241, 1999.

[3] K. R. Chaudhuri, "The basis for day and night-time control of symptoms of Parkinson's disease," European Journal of Neurology, vol. 9, supplement 3, pp. 40-43, 2002. 
[4] P. Martinez-Martin, A. H. V. Schapira, F. Stocchi et al., "Prevalence of nonmotor symptoms in Parkinson's disease in an international setting; study using nonmotor symptoms questionnaire in 545 patients," Movement Disorders, vol. 22, no. 11, pp. 1623-1629, 2007.

[5] A. M. Damiano, C. Snyder, B. Strausser, and M. K. Willian, "A review of health-related quality-of-life concepts and measures for Parkinson's disease," Quality of Life Research, vol. 8, no. 3, pp. 235-243, 1999.

[6] K. H. Karlsen, E. Tandberg, D. Arsland, and J. P. Larsen, "Health related quality of life in Parkinson's disease: a prospective longitudinal study," Journal of Neurology Neurosurgery and Psychiatry, vol. 69, no. 5, pp. 584-589, 2000.

[7] L. M. Shulman, R. L. Taback, A. A. Rabinstein, and W. J. Weiner, "Non-recognition of depression and other non-motor symptoms in Parkinson's disease," Parkinsonism \& Related Disorders, vol. 8, no. 3, pp. 193-197, 2002.

[8] K. R. Chaudhuri, L. Yates, and P. Martinez-Martin, "The non-motor symptom complex of Parkinson's disease: a comprehensive assessment is essential," Current Neurology and Neuroscience Reports, vol. 5, no. 4, pp. 275-283, 2005.

[9] K. R. Chaudhuri and K. Logishetty, "Dopamine receptor agonists and sleep disturbances in Parkinson's disease," Parkinsonism \& Related Disorders, vol. 15, supplement 4, pp. S101-S104, 2009.

[10] S. Frucht, J. D. Rogers, P. E. Greene, M. F. Gordon, and S. Fahn, "Falling asleep at the wheel: motor vehicle mishaps in persons taking pramipexole and ropinirole," Neurology, vol. 52, no. 9, pp. 1908-1910, 1999.

[11] V. Dhawan, D. G. Healy, S. Pal, and K. R. Chaudhuri, "Sleeprelated problems of Parkinson's disease," Age and Ageing, vol. 35, no. 3, pp. 220-228, 2006.

[12] M. D. Gjerstad, G. Alves, T. Wentzel-Larsen, D. Aarsland, and J. P. Larsen, "Excessive daytime sleepiness in Parkinson disease: is it the drugs or the disease?" Neurology, vol. 67, no. 5, pp. 853-858, 2006.

[13] C. L. Comella, M. Morrissey, and K. Janko, "Nocturnal activity with nighttime pergolide in Parkinson disease: a controlled study using actigraphy," Neurology, vol. 64, no. 8, pp. 14501451, 2005.

[14] B. Högl, A. Rothdach, T. C. Wetter, and C. Trenkwalder, "The effect of cabergoline on sleep, periodic leg movements in sleep, and early morning motor function in patients with Parkinson's disease," Neuropsychopharmacology, vol. 28, no. 10, pp. 1866-1870, 2003.

[15] D. J. Tompson and D. Vearer, "Steady-state pharmacokinetic properties of a 24-hour prolonged-release formulation of ropinirole: results of two randomized studies in patients with Parkinson's disease," Clinical Therapeutics, vol. 29, no. 12, pp. 2654-2666, 2007.

[16] R. Pahwa, M. A. Stacy, S. A. Factor et al., "Ropinirole 24-hour prolonged release: randomized, controlled study in advanced Parkinson disease," Neurology, vol. 68, no. 14, pp. 1108-1115, 2007.

[17] F. Stocchi, B. P. Hersh, B. L. Scott et al., "Ropinirole 24hour prolonged release and ropinirole immediate release in early Parkinson's disease: a randomized, double-blind, noninferiority crossover study," Current Medical Research and Opinion, vol. 24, no. 10, pp. 2883-2895, 2008.

[18] B. P. Hersh, N. L. Earl, R. A. Hauser, and M. Stacy, "Early treatment benefits of ropinirole prolonged release in Parkinson's disease patients with motor fluctuations," Movement Disorders, vol. 25, no. 7, pp. 927-931, 2010.
[19] GlaxoSmithKline, "Ropinirole XL prolonged-release tablets summary of product characteristics," February 2011, http: //www.medicines.org.uk/emc/medicine/20813/SPC/Requip+ $\mathrm{XL}+$ prolonged-release+tablets/.

[20] J. Reimer, M. Grabowski, O. Lindvall, and P. Hagell, "Use and interpretation of on/off diaries in Parkinson's disease," Journal of Neurology Neurosurgery and Psychiatry, vol. 75, no. 3, pp. 396-400, 2004.

[21] P. Dušek, J. Bušková, E. Růžička et al., "Effects of ropinirole prolonged-release on sleep disturbances and daytime sleepiness in Parkinson disease," Clinical Neuropharmacology, vol. 33, no. 4, pp. 186-190, 2010. 


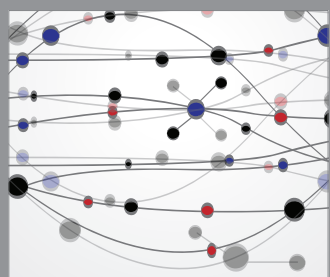

The Scientific World Journal
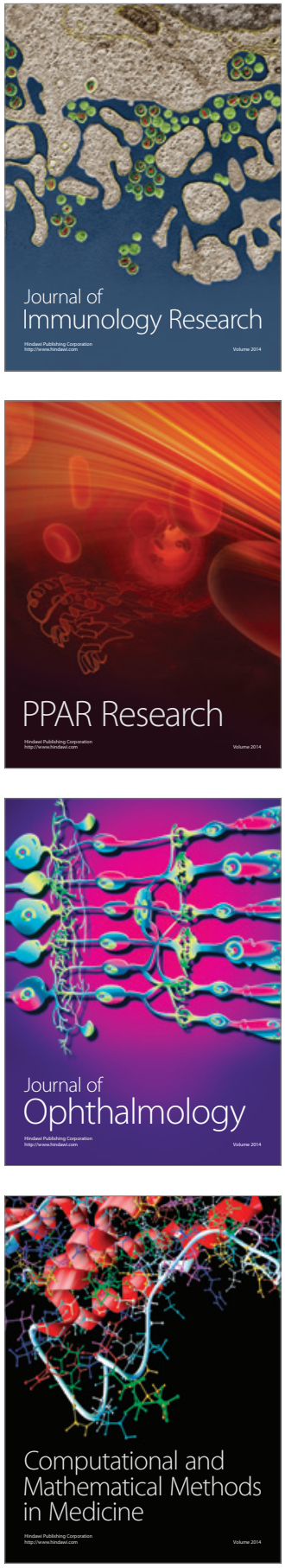

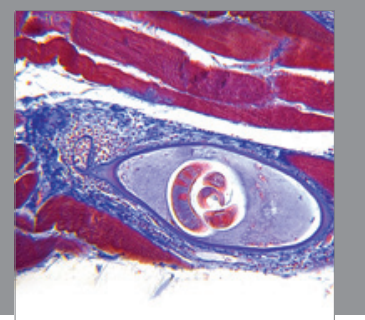

Gastroenterology

Research and Practice
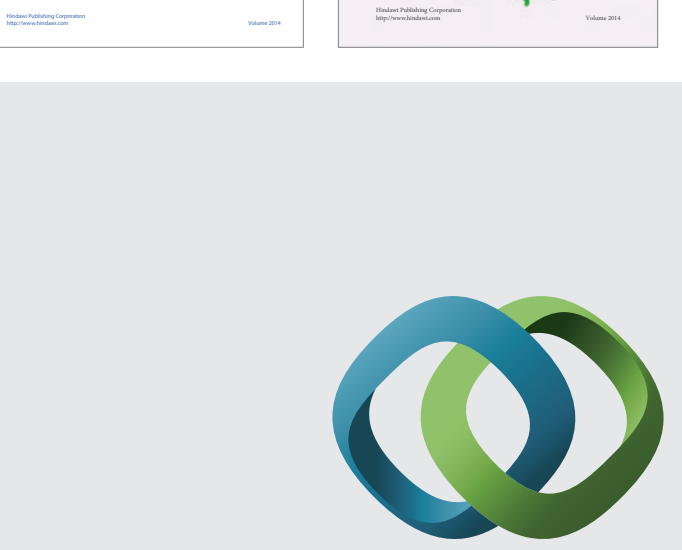

\section{Hindawi}

Submit your manuscripts at

http://www.hindawi.com
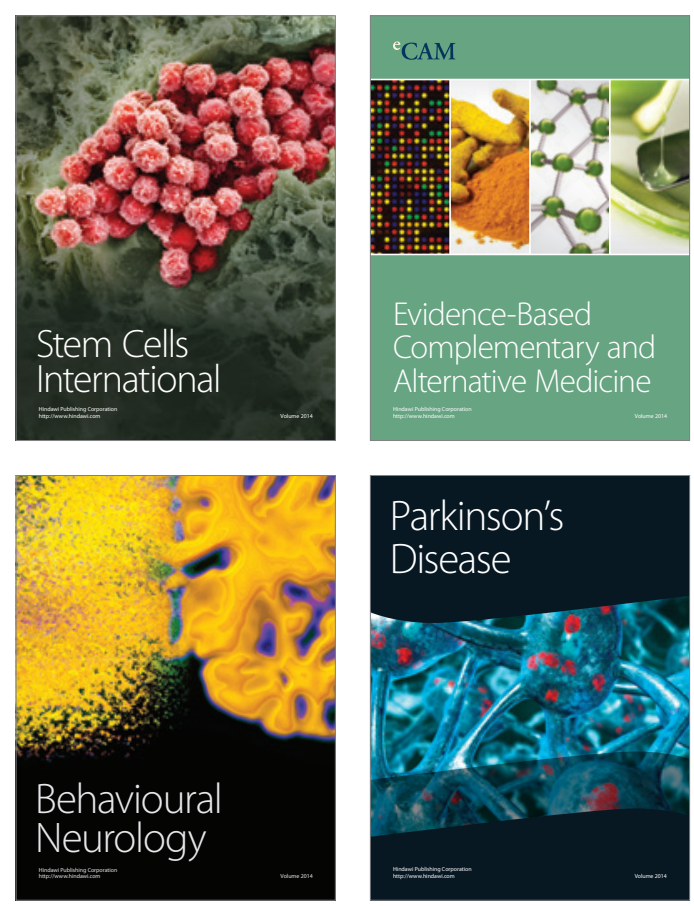

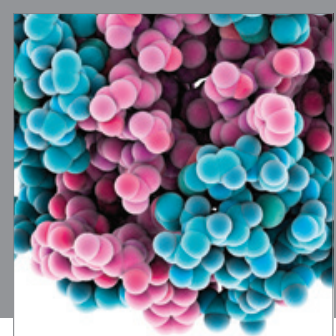

Journal of
Diabetes Research

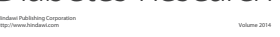

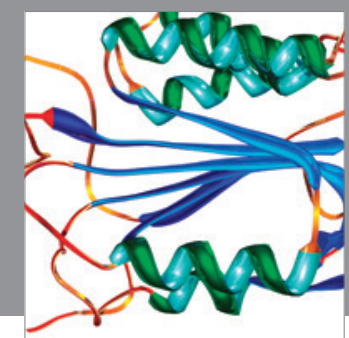

Disease Markers
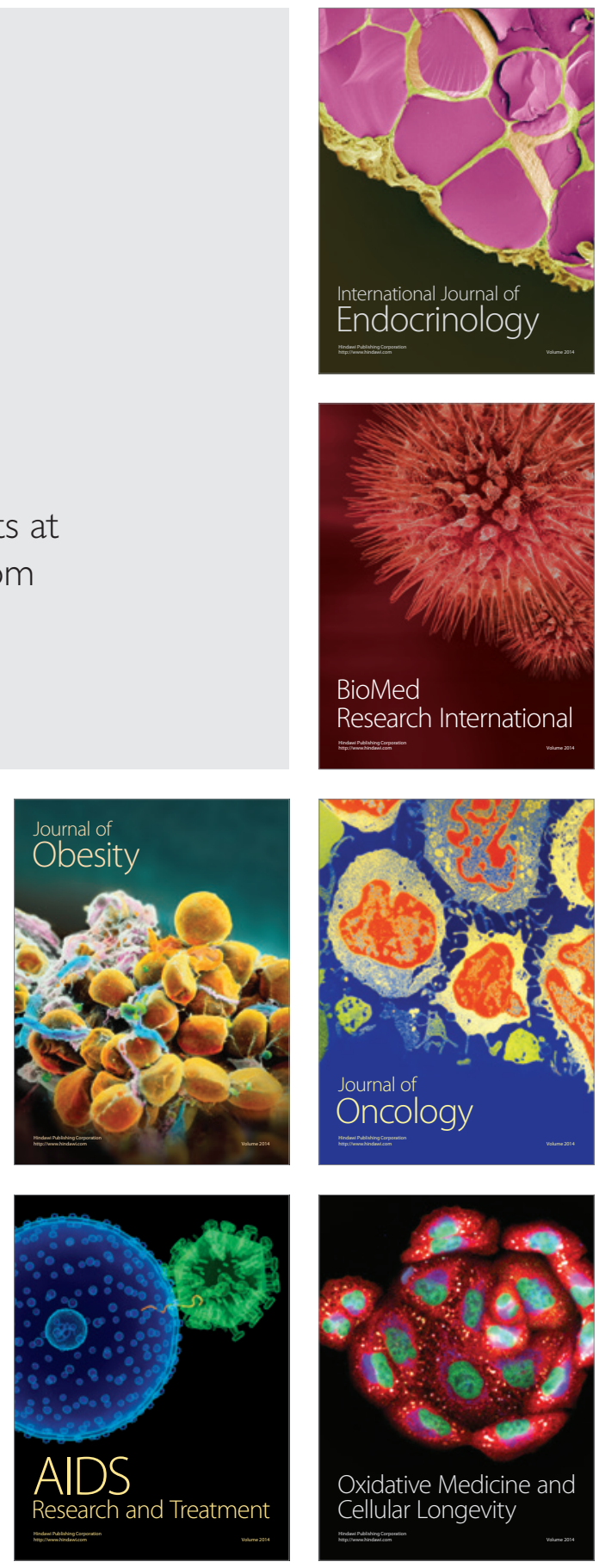